\title{
Rendimiento de cerdos alimentados con raciones conteniendo subproductos de arroz, durante la fase de crecimiento
}

\section{Performance of growing swines fed with a diet containing rice subproducts}

\author{
Victor Hurtado N, ${ }^{1 *}$ Ph.D, Rita Nobre ${ }^{2}{ }^{2}$ Ph. D, Julien Chiquieri, ${ }^{2}$ Ph.D.
}

\begin{abstract}
${ }^{1}$ Universidad de los Llanos, Escuela de Ciencias Animales, Km 12 vía Apiay, Villavicencio, Colombia. 2Universidade Estadual do Norte Fluminense. Centro de Ciências e Tecnologias Agropecuárias, Av. Alberto Lamego, 2000. Horto. Campos dos Goytacazes, RJ. Brasil. *Correspondencia: johnnie182@ hotmail.com
\end{abstract}

Recibido: Abril de 2010; Aceptado: Diciembre de 2010.

\section{RESUMEN}

Objetivo. Evaluar el rendimiento de cerdos alimentados con raciones conteniendo subproductos de arroz durante la fase de crecimiento. Materiales y métodos. Fueron utilizados 40 lechones mestizos de $31.26 \pm 4.62 \mathrm{~kg}$, distribuidos en un diseño experimental de bloques al azar, con los siguientes tratamientos 1. Control, ración basal; 2. Ración con $100 \%$ de sustitución de maíz por arroz partido; 3. Ración con $50 \%$ de sustitución de maíz por arroz partido; $4.100 \%$ de substitución de maíz por harina de arroz integral y 5 . Ración con $50 \%$ de substitución de maíz por harina de arroz integral. Resultados. En todas las fases evaluadas (I, II y fase total) fue observado un pobre desempeño de los cerdos alimentados con las raciones conteniendo harina de arroz integral, con efectos negativos más evidentes cuando el maíz fue totalmente sustituido por este subproducto (1.467 kg de consumo diario de ración y $0.512 \mathrm{~kg}$ de ganancia diaria de peso). La sustitución total o parcial de maíz por arroz partido no afectó el desempeño de los cerdos en crecimiento ( 1.681 y $1.733 \mathrm{~kg}$ de consumo diario de ración y 0.703 y $0.708 \mathrm{~kg}$ de ganancia de peso respectivamente). La peor conversión alimenticia (2.87) fue obtenida con la sustitución total del maíz por harina de arroz integral. Conclusiones. El maíz puede ser substituido en $100 \%$ en raciones para cerdos en crecimiento por arroz partido y en $50 \%$ por harina de arroz integral sin perjudicar La ganancia de peso, el consumo de ración y La conversión alimenticia.

Palabras clave: Alimentos, subproductos, arroz, harina de arroz, lechón. (Fuente: AIMS) 


\section{ABSTRACT}

Objective. To evaluate the performance of swines fed with rice sub products during the growing fase. Materials and methods. Forty hybrid piglets of $31.26 \pm 4.62 \mathrm{~kg}$, were allotted to 5 groups an experimental randomized block design: 1. Control diet; 2 . Diet plus $100 \%$ replacement of corn by broken rice; 3 . Diet plus $50 \%$ replacement of corn by broken rice; 4 . Diet plus $100 \%$ replacement of corn by whole bran rice; 5 . Diet plus $50 \%$ replacement of corn by whole bran rice. Results. Poor performance was found in all phases evaluated (I, II and total phase) in swines fed with diets containing whole bran rice. More, evident negative effects were found when corn was completely replaced for bran rice (intake food $1.467 \mathrm{~kg}$ and $0.512 \mathrm{~kg}$ of weight gain). Replacement of corn for broken rice not affect performance (intake food 1.681 and $1.733 \mathrm{~kg}$ and 0.703 and $0.708 \mathrm{~kg}$ weight gain respectively). The poorest fed conversion (2.87) was found when corn was completely replaced by whole bran rice. Conclusions. The corn can be replaced by $100 \%$ of broken rice or by $50 \%$ of whole bran rice without affect the performance of the swine during the growing phase.

Key Words: Feeds, byproducts, rice, rice flour, piglets. (Source: AIMS)

\section{INTRODUCCIÓN}

Entre los diversos ingredientes componentes de las raciones, los cereales son los que participan en mayor cantidad, contribuyendo en cerca de dos tercios del volumen total de las dietas balanceadas. La menor disponibilidad y el alto costo de las materias primas utilizadas en la preparación de raciones obligan a la búsqueda de nuevas fuentes para la alimentación de los animales. Entre esas materias primas se encuentra los partidos o quebrados de arroz y la harina de arroz integral, que son subproductos del proceso de selección e industrialización de éste cereal para el consumo humano, cuyo costo, en algunas regiones y en época de cosecha es menor al del maíz y que son poco utilizados en las raciones de animales no rumiantes.

Según los indicadores de la agropecuaria en el Brasil y en el Estado de Río de Janeiro se tuvo un área sembrada de 2.970 .000 y 2.600 hectáreas de arroz para las cosechas de 2006 - 2007, con un volumen de producción de 11.329 .200 y 9.000 toneladas respectivamente (1).

Diferentes subproductos de la industria de arroz han sido investigados para ser utilizados en la alimentación animal. Por ejemplo, el arroz partido en dietas para conejos (2), la harina de arroz desengrasada o integral para cerdos $(3,4)$ y pollos asaderos $(5,6)$, arroz con y sin cáscara para bovinos (7) y harina de arroz integral para vacas lecheras (8).

Silva et al (9) constataron incremento lineal en el consumo de ración en la medida en que se aumentaron los niveles de sustitución del maíz por arroz partido, sin embargo no hubo diferencias significativas para ganancia diaria de peso y conversión alimenticia en cerdos en crecimiento y terminación. Por otro lado, Marrero et al (10), determinaron que el maíz puede ser sustituido totalmente por arroz partido, en la dieta para cerdos en crecimiento proporcionando ganancia de peso de $720 \mathrm{~g} /$ día.

Este trabajo fue realizado con el objetivo de evaluar el efecto de los subproductos de arroz en la ración sobre el desempeño de cerdos en fase de crecimiento.

\section{MATERIALES Y METODOS}

Localización. El trabajo fue realizado en el sector de porcicultura de la Unidad de Apoyo a la Investigación del Laboratorio de Zootecnia y Nutrición animal de la Universidad Estatal del Norte Fluminense, localizada en el municipio de Campos dos 
Goytacazes, litoral norte de Río de Janeiro, Brasil.

Animales y fases experimentales. Fueron utilizados 40 lechones mestizos Landrace $x$ Large White $x$ Pietrain de 70 días de edad, con $31.26 \pm 4.62 \mathrm{~kg}$ de peso. Los semovientes se distribuyeron en un diseño experimental de bloques completos al azar, en cinco tratamientos, cuatro repeticiones y dos animales por repetición, siendo un macho y una hembra por unidad experimental. El experimento fue dividido en dos fases (Crecimiento I y Crecimiento II) de 28 días cada una.

Tratamientos. La composición centesimal de las raciones experimentales fue calculada a partir de los valores nutricionales (Tabla 1) de los ingredientes utilizando el Programa Computacional Raçao, para atender las exigencias nutricionales (Tablas 2 y 3 ) recomendadas por Rostagno (11) que corresponden a cerdos en crecimiento de 30 a $50 \mathrm{~kg}$ y de 50 a $70 \mathrm{~kg}$ respectivamente.

Las raciones experimentales constituyeron los tratamientos que fueron los siguientes:

Tratamiento 1. Ración basal, RB (utilizando maíz como principal fuente energética) o ración testigo.

Tratamiento 2. Ración con sustitución del
$100 \%$ de maíz por arroz partido, AP. Tratamiento 3. Ración con $50 \%$ de sustitución de maíz por arroz partido, AP 50.

Tratamiento 4. Ración con $100 \%$ de sustitución de maíz por harina de arroz integral, HAI.

Tratamiento 5. Ración con 50\% de sustitución de maíz por harina de arroz integral, HAI 50.

En el proceso de elaboración de las raciones experimentales los granos partidos de arroz fueron molidos para disminuir el tamaño de las partículas. Los minerales, vitaminas, aminoácidos sintéticos y sal fueron homogenizados en micromezcladora tipo $\mathrm{Y}$, posteriormente todos los ingredientes fueron incorporados en mezcladora vertical. El tiempo de mezclado de los ingredientes fue de 15 minutos.

\begin{abstract}
Alojamiento y manejo de los animales. Los animales fueron alojados en corrales de concreto de $1.8 \times 2.0$ metros, con pisos de cemento, dotados de bebederos tipo chupeta y comederos convencionales tipo canoa. La temperatura máxima y mínima durante la fase experimental fue de $25.5 \pm 1.7$ y $19.1 \pm 2.4^{\circ} \mathrm{C}$. Los animales fueron alimentados dos veces al día, a las 07:00 y 15:00 h, las sobras de alimentos en los comederos fueron recogidas y pesadas diariamente.
\end{abstract}

Tabla 1. Composición nutricional de los ingredientes utilizados en las raciones experimentales

\begin{tabular}{|c|c|c|c|c|c|c|c|c|c|}
\hline \multirow[b]{2}{*}{ Ingrediente } & \multicolumn{9}{|c|}{ Nutrientes } \\
\hline & ${ }^{1} \mathbf{P b} \%$ & $\mathrm{Ca} \%$ & Pd \% & EM Kcal/kg & $\mathrm{Na} \%$ & Lis \% & Met \% & Tre \% & Fibra \% \\
\hline Maíz² & 8.26 & 0.03 & 0.08 & 3340 & 0.02 & 0.19 & 0.15 & 0.26 & 1.73 \\
\hline Torta de soya ${ }^{2}$ & 45.32 & 0.24 & 0.18 & 3154 & 0.02 & 2.53 & 0.59 & 1.55 & 5.41 \\
\hline Arroz Partido ${ }^{2}$ & 8.47 & 0.04 & 0.05 & 3491 & 0.02 & 0.25 & 0.16 & 0.23 & 0.55 \\
\hline \multicolumn{10}{|l|}{ Harina de } \\
\hline arroz integral ${ }^{2}$ & 13.24 & 0.01 & 0.32 & 3111 & 0.04 & 0.46 & 0.19 & 0.35 & 7.88 \\
\hline Fosfato bicálcico ${ }^{2}$ & & 24.50 & 18.50 & & & & & & \\
\hline \multicolumn{10}{|l|}{ Carbonato de } \\
\hline Calcio $^{2}$ & & 38.40 & & & & & & & \\
\hline Aceite de soya ${ }^{2}$ & & & & 8300 & & & & & \\
\hline $\mathrm{Sal}^{2}$ & & & & & 39.70 & & & & \\
\hline L-Lisina $\mathrm{HCl}^{3}$ & & & & & & 78.50 & & & \\
\hline Metionina $^{3}$ & & & & & & & 99.00 & & \\
\hline Treonina $^{3}$ & & & & & & & & 78.00 & \\
\hline
\end{tabular}

${ }^{1} \mathrm{~Pb}$, Proteína bruta; Ca, calcio; Pd, Fósforo disponible; EM, energía Metabolizable; Na, sodio; Lis, lisina; Met, metionina; Tre, treonina. ${ }^{2}$ Rostagno (11). ${ }^{3}$ Concentración del ingrediente activo del producto comercial. 
Tabla 2. Composición centesimal de la ración para cerdos en fase I de crecimiento.

\begin{tabular}{|c|c|c|c|c|c|}
\hline \multirow[b]{2}{*}{ Ingrediente } & \multicolumn{5}{|c|}{ Tratamientos } \\
\hline & RB & AP & AP50 & HAI & HAI50 \\
\hline Maíz & 72.437 & 0.000 & 36.218 & 0.000 & 36.218 \\
\hline Torta de soya & 24.073 & 23.400 & 23.560 & 13.810 & 18.760 \\
\hline Arroz partido & 0.000 & 73.793 & 37.416 & 0.000 & 0.000 \\
\hline Harina de arroz integral & 0.000 & 0.000 & 0.000 & 80.051 & 40.280 \\
\hline Fosfato bicálcico & 1.249 & 1.365 & 1.310 & 0.276 & 0.760 \\
\hline Carbonato de calcio & 0.640 & 0.550 & 0.594 & 1.172 & 0.907 \\
\hline Aceite de soya & 0.703 & 0.000 & 0.000 & 3.690 & 2.120 \\
\hline Vitaminas $^{1}$ & 0.150 & 0.150 & 0.150 & 0.150 & 0.150 \\
\hline Minerales $^{2}$ & 0.100 & 0.100 & 0.100 & 0.100 & 0.100 \\
\hline Sal yodada & 0.405 & 0.405 & 0.405 & 0.366 & 0.385 \\
\hline $\mathrm{L}$ - Lisina $\mathrm{HCl}$ & 0.189 & 0.151 & 0.174 & 0.226 & 0.212 \\
\hline Metionina & 0.018 & 0.063 & 0.016 & 0.036 & 0.028 \\
\hline Treonina & 0.026 & 0.042 & 0.047 & 0.113 & 0.072 \\
\hline $\mathrm{BHT}$ & 0.010 & 0.010 & 0.010 & 0.010 & 0.010 \\
\hline \multicolumn{6}{|l|}{ Composición calculada } \\
\hline Proteína bruta & 16.850 & 16.850 & 16.830 & 16.850 & 16.820 \\
\hline EM kcal / kg & 3235 & 3314 & 3258 & 3230 & 3230 \\
\hline P disponible & 0.332 & 0.332 & 0.332 & 0.332 & 0.332 \\
\hline Calcio & 0.631 & 0.631 & 0.631 & 0.631 & 0.631 \\
\hline Lisina digestible & 0.895 & 0.895 & 0.895 & 0.895 & 0.895 \\
\hline Metionina & 0.269 & 0.269 & 0.269 & 0.269 & 0.269 \\
\hline Treonina & 0.582 & 0.582 & 0.582 & 0.582 & 0.582 \\
\hline Sodio & 0.180 & 0.180 & 0.180 & 0.180 & 0.180 \\
\hline Fibra bruta ${ }^{3}$ & 2.556 & 1.672 & 2.107 & 7.055 & 4.810 \\
\hline Composición Estimada $\mathrm{PB} \%{ }^{3}$ & 17.48 & 16.42 & 16.92 & 16.27 & 16.83 \\
\hline
\end{tabular}

${ }^{1}$ Niveles de garantía por kg de producto: Biotina 16.56 mg; Vit. E 10.500 mg; Piridoxina 700 mg; Vit. K3 2.800 mg; Colina 126 g; Niacina 13.650 mg; Acido Pantoténico 7.350 mg; Vit. A 2.800 UI; Tiamina 700 mg; Vit. B12 11.550 mcg; Vitamina D3 1.050 UI; Acido Fólico 420 mg; Riboflavina 2.100 mg; Antioxidante 1.500 mg; Se, 136 mg; ${ }^{2}$ Niveles de garantía por kg de producto: Fe, $45.000 \mathrm{mg}$; Cu, 37. 000 mg; Mn, 25.000 mg; Co, 300 mg; I, 800 mg; Se, 120 mg. ${ }^{3}$ Análisis realizado en el Laboratorio de Nutrición Animal, Centro de Ciencias y Tecnologías Agropecuarias, Universidad Estatal del Norte Fluminense, UENF.

Los cerdos fueron pesados al inicio y final de cada fase evaluada, en total se realizaron tres pesajes a los 0,28 y 56 días del experimento, es decir cuando los animales tenían 70, 98 y 126 días de edad.

Análisis estadístico. Las variables analizadas incluyeron consumo diario de ración, ganancia diaria de peso y conversión alimenticia. Los datos fueron sometidos a análisis de varianza, siendo las medias comparadas por la prueba de Tukey a $5 \%$ de probabilidad.
El modelo estadístico utilizado fue el siguiente:

$Y_{i j}=\mu+T i+\beta j+\varepsilon i j$ donde

$Y i j=$ Observación $Y$ en el tratamiento $i$, en el bloque $\mathrm{j}$.

$\mu=$ Media general.

$\mathrm{Ti}=$ Efecto del tratamiento $\mathrm{i}$, siendo $\mathrm{i}$ : $1,2,3,4$ y 5 (sustitución de maíz por subproductos de arroz).

$\beta j=$ Efecto del bloque $j$, siendo $j: 1,2,3$ y 4. 
Tabla 3. Composición centesimal de la ración para cerdos en fase II de Crecimiento

\begin{tabular}{|c|c|c|c|c|c|}
\hline \multirow[b]{2}{*}{ Ingrediente } & \multicolumn{5}{|c|}{ Tratamientos } \\
\hline & RB & AP & AP50 & HAI & HAI50 \\
\hline Maíz & 77.290 & 0.00 & 38.645 & 0.000 & 38.645 \\
\hline Torta de soya & 19.959 & 19.470 & 19.700 & 9.210 & 14.570 \\
\hline Arroz partido & 0.000 & 77.990 & 39.120 & 0.000 & 0.000 \\
\hline Harina de arroz integral & 0.000 & 0.000 & 0.000 & 85.051 & 42.593 \\
\hline Fosfato bicálcico & 0.996 & 1.122 & 1.060 & 0.000 & 0.476 \\
\hline Carbonato de calcio & 0.615 & 0.515 & 0.565 & 1.157 & 0.900 \\
\hline Aceite de soya & 0.223 & 0.00 & 0.000 & 3.565 & 1.850 \\
\hline Vitaminas $^{1}$ & 0.150 & 0.150 & 0.150 & 0.150 & 0.150 \\
\hline Minerales ${ }^{2}$ & 0.100 & 0.100 & 0.100 & 0.100 & 0.100 \\
\hline Sal yodada & 0.379 & 0.380 & 0.380 & 0.337 & 0.358 \\
\hline L - Lisina $\mathrm{HCl}$ & 0.226 & 0.180 & 0.203 & 0.261 & 0.243 \\
\hline Metionina & 0.015 & 0.009 & 0.012 & 0.033 & 0.024 \\
\hline Treonina & 0.037 & 0.074 & 0.055 & 0.126 & 0.081 \\
\hline $\mathrm{BHT}$ & 0.010 & 0.010 & 0.010 & 0.010 & 0.010 \\
\hline \multicolumn{6}{|l|}{ Composición calculada } \\
\hline Proteína bruta & 15.43 & 15.43 & 15.43 & 15.43 & 15.43 \\
\hline EM kcal / kg & 3230 & 3336 & 3277 & 3232 & 3228 \\
\hline P disponible & 0.282 & 0.282 & 0.282 & 0.282 & 0.282 \\
\hline Calcio & 0.551 & 0.551 & 0.551 & 0.551 & 0.551 \\
\hline Lisina digestible & 0.829 & 0.829 & 0.829 & 0.829 & 0.829 \\
\hline Metionina & 0.249 & 0.249 & 0.249 & 0.249 & 0.249 \\
\hline Treonina & 0.539 & 0.539 & 0.359 & 0.539 & 0.539 \\
\hline Sodio & 0.170 & 0.170 & 0.170 & 0.170 & 0.170 \\
\hline Fibra bruta ${ }^{3}$ & 2.417 & 1.482 & 1.949 & 7.200 & 4.813 \\
\hline Composición Estimada $\mathrm{PB} \%{ }^{3}$ & 16.22 & 15.08 & 15.68 & 15.77 & 15.60 \\
\hline
\end{tabular}

${ }^{1}$ Niveles de garantía por kg de producto: Biotina 16.56 mg; Vit. E 10.500 mg; Piridoxina 700 mg; Vit. K3 2.800 mg; Colina 126 g; Niacina 13.650 mg; Acido Pantoténico 7.350 mg; Vit. A 2.800 UI; Tiamina 700 mg; Vit. B12 11.550 mcg; Vitamina D3 1.050 UI; Acido Fólico 420 mg; Riboflavina 2.100 mg; Antioxidante 1.500 mg; Se, 136 mg; ${ }^{2}$ Niveles de garantía por kg de producto: Fe, 45.000 mg; Cu, 37. 000 mg; Mn, 25.000 mg; Co, 300 mg; I, 800 mg; Se, 120 mg. ${ }^{3}$ Análisis realizado en el Laboratorio de Nutrición Animal, Centro de Ciencias y Tecnologías Agropecuarias, Universidad Estatal del Norte Fluminense, UENF.

\section{RESULTADOS}

Los resultados de consumo diario de ración, ganancia diaria de peso y conversión alimenticia de lechones en crecimiento son presentados en las tablas 4,5 y 6.

En la fase I $(30-50 \mathrm{~kg})$ de crecimiento la harina de arroz integral en sustitución total al maíz afectó negativamente $(p<0.05)$ la ganancia diaria de peso de los animales, hubo un incremento significativo $(p<0.05)$ en el consumo de la ración conteniendo $50 \%$ de arroz partido en relación con la ración con sustitución total del maíz por
Tabla 4. Desempeño de lechones alimentados con raciones conteniendo subproductos de arroz en fase I (30-50 kg) de crecimiento.

\begin{tabular}{lcccccc}
\hline \multirow{2}{*}{ Variable } & \multicolumn{6}{c}{ Tratamientos } \\
\cline { 2 - 7 } & RB & AP & AP50 & HAI & HAI50 & CV \\
\hline Peso inicial, $\mathrm{kg}$ & 32.325 & 30.100 & 31.600 & 31.525 & 30.765 & 8.6 \\
Peso final, $\mathrm{kg}$ & 50.917 & 49.784 & 50.248 & 46.337 & 48.657 & 7.6 \\
$\begin{array}{l}\text { Consumo diario } \\
\text { de ración, kg }\end{array}$ & $1.603^{\mathrm{ab}}$ & $1.663^{\mathrm{ab}}$ & $1.686^{\mathrm{a}}$ & $1.467^{\mathrm{b}}$ & $1.523^{\mathrm{ab}}$ & 8.7 \\
$\begin{array}{l}\text { Ganancia diaria } \\
\text { de peso, } \mathrm{kg}\end{array}$ & $0.664^{\mathrm{a}}$ & $0.703^{\mathrm{a}}$ & $0.666^{\mathrm{a}}$ & $0.529^{\mathrm{b}}$ & $0.639^{\mathrm{ab}}$ & 13.6 \\
$\begin{array}{l}\text { Conversión } \\
\text { alimenticia }\end{array}$ & 2.41 & 2.36 & 2.53 & 2.77 & 2.38 & 14.9 \\
\hline
\end{tabular}

Letras distintas en la misma línea indican diferencia significativa $(p<0.05)$ por la prueba de Tukey 
Tabla 5. Desempeño de lechones alimentados con raciones conteniendo subproductos de arroz en fase II (50$70 \mathrm{~kg}$ ) de crecimiento

\begin{tabular}{lcccccc}
\hline \multirow{2}{*}{ Variable } & \multicolumn{5}{c}{ Tratamientos } \\
\cline { 2 - 7 } & RB & AP & AP50 & HAI & HAI50 & CV \\
\hline Peso inicial, $\mathrm{kg}$ & 50.917 & 49.784 & 50.248 & 46.337 & 48.657 & 7.6 \\
Peso final, $\mathrm{kg}$ & 69.565 & 69.440 & 71.220 & 60.197 & 64.925 & 6.4 \\
Consumo diario de ración, $\mathrm{kg}$ & $1.750^{\mathrm{a}}$ & $1.697^{\mathrm{a}}$ & $1.779^{\mathrm{a}}$ & $1.467^{\mathrm{b}}$ & $1.534^{\mathrm{b}}$ & 6.7 \\
Ganancia diaria de peso, $\mathrm{kg}$ & $0.666^{\mathrm{ab}}$ & $0.702^{\mathrm{a}}$ & $0.749^{\mathrm{a}}$ & $0.495^{\mathrm{c}}$ & $0.581^{\mathrm{bc}}$ & 10.5 \\
Conversión alimenticia & $2.63^{\mathrm{ab}}$ & $2.42^{\mathrm{a}}$ & $2.38^{\mathrm{a}}$ & $2.96^{\mathrm{b}}$ & $2.64^{\mathrm{b}}$ & 13.1 \\
\hline Letras distintas en la misma línea indican diferencia \\
significativa $(\mathrm{p}<0.05$ ) por la prueba de Tukey
\end{tabular}

la harina de arroz integral. Entretanto, la conversión alimenticia no fue influenciada por los subproductos de arroz.

El consumo diario de ración y la ganancia diaria de peso de los cerdos alimentados con raciones en la cual el maíz fue sustituido en forma parcial o total por arroz partido y sustitución del $50 \%$ por harina de arroz integral muestran resultados estadísticamente semejantes a la ración basal.

En la fase II (50-70 kg) de crecimiento la sustitución parcial y total de maíz por harina de arroz integral redujo $(p<0.05)$ el consumo diario de ración (Tabla 5 ). La ración con $100 \%$ de sustitución de maíz por harina de arroz integral disminuye la ganancia diaria de peso $(p<0.05)$ en relación a los cerdos alimentados con raciones conteniendo maíz y arroz partido como fuente energética.

Tabla 6. Desempeño de lechones alimentados con raciones conteniendo subproductos de arroz en fase total de crecimiento $(30-70 \mathrm{~kg})$.

\begin{tabular}{lcccccc}
\hline \multirow{2}{*}{ Variable } & \multicolumn{5}{c}{ Tratamientos } \\
\cline { 2 - 7 } & RB & AP & AP50 & HAI & HAI50 & CV \\
\hline Peso inicial, $\mathrm{kg}$ & 32.325 & 30.100 & 31.600 & 31.525 & 30.765 & 7.6 \\
Peso final, $\mathrm{kg}$ & 69.565 & 69.440 & 71.220 & 60.197 & 64.925 & 6.4 \\
Consumo diario de ración, $\mathrm{kg}$ & $1.676^{\mathrm{a}}$ & $1.681^{\mathrm{a}}$ & $1.733^{\mathrm{a}}$ & $1.467^{\mathrm{b}}$ & $1.529^{\mathrm{b}}$ & 6.1 \\
Ganancia diaria de peso, $\mathrm{kg}$ & $0.665^{\mathrm{bb}}$ & $0.703^{\mathrm{a}}$ & $0.708^{\mathrm{a}}$ & $0.512^{\mathrm{C}}$ & $0.610^{\mathrm{b}}$ & 8.84 \\
Conversión alimenticia & $2.51^{\mathrm{a}}$ & $2.39^{\mathrm{a}}$ & $2.45^{\mathrm{a}}$ & $2.87^{\mathrm{b}}$ & $2.51^{\mathrm{a}}$ & 9.1 \\
\hline
\end{tabular}

Letras distintas en la misma línea indican diferencia significativa $(p<0.05)$ por la prueba de Tukey
Los cerdos alimentados con raciones conteniendo arroz partido presentan mejor conversión alimenticia $(p<0.05)$, que los alimentados con ración con sustitución total del maíz por harina de arroz integral, la conversión alimenticia de los cerdos alimentados con ración con remplazo parcial del maíz por arroz partido en la segunda fase mejoró con respecto a la fase I de crecimiento. Los resultados de la segunda fase evidencian los efectos negativos de la sustitución total de maíz por la harina de arroz integral para cerdos en crecimiento, como fue observado en la primera fase de esta investigación.

Cuando los resultados de las fases I y II de crecimiento fueron analizados en conjunto (Tabla 6), el desempeño de los animales fue semejante al observado en cada fase por separado. La sustitución parcial o total de maíz por harina de arroz integral redujo significativamente el consumo diario de ración $(p<0.05)$. Los cerdos de los tratamientos en los cuales el maíz fue reemplazado por el arroz partido presentaron mayor ganancia de peso diario $(p<0.05)$, cuando fueron comparados con los tratamientos conteniendo harina de arroz integral.

En la figura 1 se ilustra el efecto de los subproductos de arroz sobre el peso total de los cerdos al inicio y final de los dos periodos experimentales

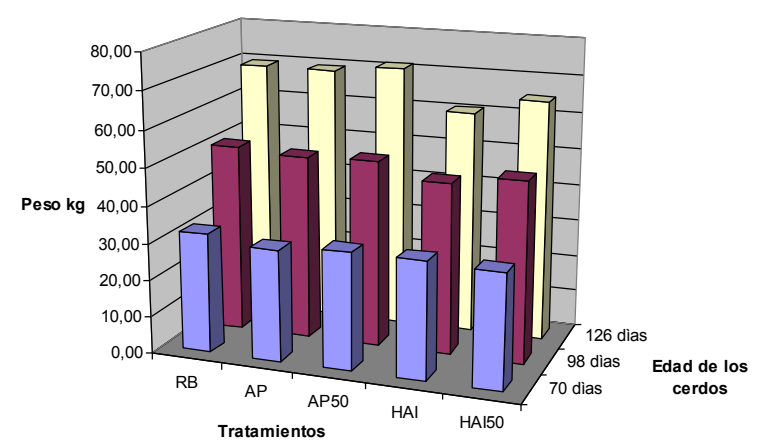

Figura 1. Peso total de los cerdos al inicio y final de cada fase experimental 
Del mismo modo, el peor resultado de conversión alimenticia ocurrió cuando los animales fueron alimentados con raciones con $100 \%$ de sustitución de maíz por harina de arroz integral. En términos relativos el reemplazo total de maíz por harina de arroz integral representa reducción en $23.0,27.1$ y $27.6 \%$ de la ganancia de peso diario durante la fase total de crecimiento en relación con los tratamiento testigo y sustitución de 100 y $50 \%$ de maíz por arroz partido respectivamente.

\section{DISCUSIÓN}

En la fase I de crecimiento (30-50 kg) la menor ganancia de peso observada en los cerdos alimentados con ración conteniendo $100 \%$ de sustitución de maíz por harina de arroz integral está relacionada con el contenido relativamente alto de fibra de este ingrediente (11), que resultó en ración con un valor calculado de fibra bruta de $7.06 \%$ (Tabla 2), comprometiendo de esta forma el consumo y aprovechamiento adecuado de nutrientes para maximizar el desempeño de los cerdos en esta fase. Además de esto, la fibra reduce el tiempo de exposición de los nutrientes a la superficie de absorción en el tracto gastrointestinal, disminuyendo de esta forma el aprovechamiento de los mismos.

El mejor desempeño de los animales alimentados con raciones conteniendo arroz partido puede ser explicado por la composición nutricional y la digestibilidad de los nutrientes contenidos en el arroz partido, que en el caso de los aminoácidos esenciales, la digestibilidad aparente del arroz partido es superior al $81 \%$ (12). Se observa también, que la sustitución de $50 \%$ del maíz por harina de arroz integral no afectó negativamente la ganancia de peso de los animales sugiriendo que niveles de fibra bruta en la ración hasta de $4.8 \%$, no comprometen esta variable.
Los resultados de cerdos en crecimiento (Fases I y II) alimentados con raciones conteniendo diferentes niveles de subproductos de arroz, sugieren que la reducción del consumo en las raciones con harina de arroz integral resulta en menor disponibilidad de nutrientes y de energía para obtener mayor ganancia de peso.

La mejoría en la conversión alimenticia del tratamiento con sustitución parcial del maíz por arroz partido en la fase experimental II en relación con la fase anterior se explica por un mejor aprovechamiento de los nutrientes debido al ligero incremento en los valores de proteína estimados respecto a los calculados que favorecen mayor formación de tejido en esta fase de desarrollo.

Marrero et al (10) encontraron valores semejantes trabajando con cerdos de peso inicial de $24 \mathrm{~kg}$, alimentados con niveles crecientes de arroz partido durante las fases de crecimiento y terminación, en una fase experimental de 90 días.

La reducción del consumo de ración en los tratamientos con harina de arroz integral está relacionada con la consistencia y densidad de la dieta que produce sensación de saciedad en los animales, que no permite la disponibilidad de la cantidad de nutrientes necesarios para un optimo desempeño.

Los resultados obtenidos en esta investigación coinciden con los encontrados por Silva et al (9) que no encontraron efectos significativos de la sustitución parcial y total del maíz por arroz partido sobre el desempeño zootécnico de cerdos en crecimiento, constatando que el arroz partido puede sustituir totalmente al maíz. 
En conclusión, el arroz partido puede ser incluido hasta en $100 \%$ y la harina de arroz integral hasta en $50 \%$ en sustitución al maíz en raciones para cerdos en crecimiento de los 30 a los $70 \mathrm{~kg}$ de peso vivo sin perjudicar el desempeño zootécnico.

\section{REFERENCIAS}

1. Companhia Nacional de Abastecimento, CONAB. Comparativo de área produtividade e produção safras 2005/2006 e 2006/2007. Brasilia: Companhia Nacional de Abastecimento; 2007.

2. Brum Jr, BS, Quirera de arroz na dieta de frangos de Corte e coelhos em crescimento. [Tese de mestrado], Santa María:Universidad Federal de Santamaría; 2006.

3. Wang JF, Jensen BB, Jorgensen $H$, Li DF, Lindberg JE. Ileal and total tract digestibility, and protein and fat balance in pigs fed rice with addition of potato starch, sugar beet pulp or wheat bran. Anim Feed Sci Technol 2002; 102:125-136.

4. Moreira JA, Vitti DMSS, Neto MAT, Lopes JB. Phytase enzyme in diets containing defatted rice bran for growing swine. Sci Agric 2003; 60:631-636.

5. Conte AJ, Teixeira AS, Fialho ET, Schoulten NA, Bertechini AG. Efeito da fitase e xilanase sobre o desempenho e as características ósseas de frangos de corte alimentados com dietas contendo farelo de arroz. Br J Anim Sci 2003; 32:1147-1156.
6. Brum Jr BS, Zanella I, Toledo GSP, Xavier EG, Vieira TA, Gonçalves EC, Brum H, Oliveira JLS. Dietas para frangos de corte contendo quirera de arroz. Cienc Rural 2007; 37:1423-1429.

7. Marichal, MJ, Carriquiry, M, Trujillo, AI. Degradabilidad de la materia seca de subproductos del arroz, del maíz y de descartes hortifrutícolas. En: Memorias XXI Reunión Asociación Latinoamericana de Producción Animal; 2009, San Juan, Puerto Rico. San Juan: ALPA; 2009.

8. Nörnberg JL, Júnior WS, López J, Costa PB. Valor do farelo de arroz integral como fonte de gordura na dieta de vacas Jersey na fase inicial de lactação: Digestibilidade aparente de nutrientes. Br J Anim Sci 2004; 33(Supl. 3):2412-2421

9. Silva HO, Menezes IC, Braga DF, Schoulten, NA, Silva, LF. Quirera de arroz em substitução ao milho em rações para suínos em crescimento e terminação: Desempenho e características de carcaça. Rev Cient Prod Anim 2004; 6:24-32. 
10. Marrero LI, Silveira E, García EL. Evaluación de sistemas de alimentación porcina a partir de fuentes energéticas de producción nacional. Rev Electrón Vet; 2005 (fecha de acceso 15 de diciembre de 2009). 6:6. URL Disponible en http://veterinaria.org/revistas/ redvet/n060605.html

11. ROSTAGNO HS. Tabelas Brasileiras para aves e suínos. Composição de alimentos e exigências nutricionais. 2a ed. Viçosa: Universidade federal de Viçosa; 2005; 186.
12. Apolônio LR, Donzele JL, Oliveira RFM, Souza AVC, Silva FCO, Bünzen S. 2003. Digestibilidade Ileal de Aminoácidos de alguns Alimentos, Determinada pela Técnica da Cânula T Simples com Suínos. Br J Anim Sci 2003, 32:605-614 